\title{
Opinion Consensus of Modified Hegselmann-Krause Models
}

\author{
Yuecheng Yang, Dimos V. Dimarogonas and Xiaoming Hu
}

\begin{abstract}
We consider the opinion consensus problem using a multi-agent setting based on the Hegselmann-Krause (H-K) Model. Firstly, we give a sufficient condition on the initial opinion distribution so that the system will converge to only one cluster. Then, modified models are proposed to guarantee convergence for more general initial conditions. The overall connectivity is maintained with these models, while the loss of certain edges can occur. Furthermore, a smooth control protocol is provided to avoid the difficulties that may arise due to the discontinuous right-hand side in the H-K model.
\end{abstract}

\section{INTRODUCTION}

The opinion consensus problem is about opinion compromise of a certain event by different agents. Assume that the opinion is continuous, and that all agents have bounded confidence in the way that they only consider the opinions that are close to their own opinion. Agent-based models of opinion dynamics under these assumptions have been established in the beginning of this century by Hegselmann and Krause [1] and Weisbuch et al [2]. Both models lead to clustering of opinions in a similar way. In this paper we will consider the model of Hegselmann and Krause (H-K).

The previous study about the $\mathrm{H}-\mathrm{K}$ model shows that not all initial positions corresponding to a connected graph will lead to consensus [3], [4], [8]. This is because during the process the graph can become disconnected since the neighborhood is based on opinion differences between pairs of agents. The loss of connectivity can yield several clusters of agent opinions in different positions. This phenomenon is also observed in the initial paper by Hegselmann and Krause [1]. However, even for one-dimensional case, few theoretical results have been obtained so far regarding the relationship between this loss of connectivity and the initial opinion distribution. Although the H-K model can be easily extended to higher dimensional spaces, this paper will mainly focus on the one-dimensional case.

On the other hand, instead of imposing constraints on the initial distribution, one can modify the model to guarantee that consensus is achieved for any initial opinions. This is related to the connectivity maintenance problem in the multi-agent system theory. A way to achieve this is by using potential functions. The main idea is that the force between two agent opinions becomes infinitely large when the difference between the opinions becomes big enough, i.e., near the boundary of confidence. This approach has been used by several researchers in the past few years, e.g.,

Yuecheng Yang and Xiaoming Hu are with the Optimization and Systems Theory division, Department of Mathematics, Royal institute of Technology (KTH), 10044 Stockholm, Sweden. Dimos Dimarogonas is with automatic control lab, School of Electrical Engineering, KTH. This work is supported by SSF, the Swedish Research Council and the EU HYCON 2 NoE.
[11], [12]. Bounded controllers for connectivity control are considered in [10]. The common idea in these papers is that no edge is allowed to break during the process, thus imposing constraints in the relative states of pairs of agents that constitute an edge. However, this is only a sufficient condition for connectivity maintenance because the loss of some "non-crucial" edges may not influence the connectivity. In this paper we use topological arguments to guarantee connectivity instead of applying infinite potentials when an edge is bound to break. In particular, inspired by the idea used in [13], we show that common neighbors play an important role in the problem. If two nodes $i$ and $j$ such that $(i, j) \in E$ have some common neighbors, then the edge $(i, j)$ can be allowed to break because they are still connected by the common neighbors. On the contrary, if they do not have any common neighbors, then the edge becomes important and should not break. This approach seems more intuitive from a social networks perspective since it is more natural to take into account the number of common neighbors than applying infinite forces to maintain the graph connected.

The modified model that we provide in this paper guarantees opinion consensus for almost any connected initial opinion distribution, even if the ratio between the opinion diversity and the confidence bound is significant. Usually one obtains clustering behavior, i.e., disconnectedness, of the original H-K model when this ratio is big. This issue is overcome by using the modified model in Section III. An requirement of our model is that two or more agents cannot have the same initial opinions. Since this is a possible scenario in the real world, we provide another model to deal with this case. Furthermore, for the original H-K model, the right-hand side is not a continuous function of the state $x$. This results to measure zero sets of initial conditions from which the solution may not be unique. We introduce in the paper a smooth modification of the model in order to avoid this.

The remainder of the paper is summarized as follows: in Section II we formulate the problem under consideration. The modified version of the $\mathrm{H}-\mathrm{K}$ model is presented and analyzed in Section III. A smoothed version of this is provided in Section IV. Section V includes simulations that support the derived theoretical results. Finally, a summary of the results of this paper as well as possible directions of future work are included in Section VI.

\section{MATHEMATICAL PRELIMINARIES}

\section{A. Basic concepts from graph theory}

In this section, we review some concepts from graph theory that will be used in this paper. These definitions can 
be otherwise found in a standard textbook on graph theory.

Consider a set of $n$ nodes denoted by $V=\{1,2, \ldots, n\}$ and a subset $E \subset V \times V$. We call $G=(V, E)$ is a graph with the set of vertices (or nodes) $V$ and the set of edges $E$.In $G=(V, E)$, the neighbor set of the vertex $i$ is defined by

$$
\mathscr{N}_{i}=\{j \in V \mid(j, i) \in E\} .
$$

A graph $G=(V, E)$ is called undirected if $(i, j) \in E$ implies $(j, i) \in E$. In an undirected graph, if there is an edge connecting two vertices, i.e., $(i, j) \in E$, then these two vertices $i$, $j$ are called adjacent. A graph is called complete if any two nodes are adjacent. A path from a vertex $i$ to another vertex $j$ is a sequence of distinct vertices starting with $i$ and ending with $j$, in which each vertex is adjacent to its next vertex. Two vertices $i$ and $j$ are called connected if there exists a path from $i$ to $j$. An undirected graph is call a connected graph if any pair of vertices is connected in it.

\section{B. Introduction of Hegselmann-Krause model}

Consider a system of $n$ autonomous agents labeled as $1,2, \ldots, n$, whose opinions are located in the one-dimensional Euclidean space $\mathbb{R}$. We denote the set of all agents as $V=\{1,2, \ldots, n\}$. For an agent $i \in V$, the position of it's opinion is denoted by $x_{i}(t) \in \mathbb{R}$, which has the following dynamics:

$$
\dot{x}_{i}(t)=u_{i}(t),
$$

where $u_{i}(t)$ is considered as the controller of agent $i$. The consensus problem is to find the controllers $u_{i}(t)$ so that the

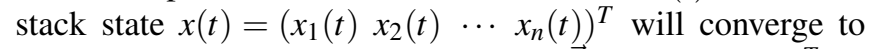
the subspace generated by the vector $\overrightarrow{1}=\left(\begin{array}{llll}1 & 1 & \cdots & 1\end{array}\right)^{T}$ as $t \rightarrow \infty$.

If the edge set $E \subset V \times V$ is given, one can then define a graph $G=(V, E)$ and generate a basic control protocol for the consensus problem:

$$
\dot{x}_{i}(t)=u_{i}(t)=\sum_{j \in \mathscr{N}_{i}}\left(x_{j}(t)-x_{i}(t)\right)
$$

It is a well-known fact [7] that the system (3) will converge to the equilibrium $x_{i}(t)=\alpha, i=1,2, \ldots, n$ if the graph $G$ is connected, where $\alpha=\frac{1}{n} \sum_{i=1}^{n} x_{i}(0)$.

Now assume that the graph $G=(V, E)$ is defined by $V=$ $\{1,2, \ldots, n\}$ and $E=\left\{(i, j)|| x_{i}-x_{j} \mid \leq d\right\}$ for some $d>0$. Applying the same control in (3) with this definition of the graph, we obtain the Hegselmann-Krause (H-K) model:

$$
\dot{x}_{i}(t)=\sum_{j:\left|x_{j}-x_{i}\right| \leq d}\left(x_{j}(t)-x_{i}(t)\right) .
$$

From now on, we will call $G=(V, E)$ the corresponding graph of $x(t)$, where $V=\{1,2, \ldots, n\}$ and $E=\left\{(i, j) \| x_{i}(t)-\right.$ $\left.x_{j}(t) \mid \leq d\right\}$. Since connectivity can change as the system evolves, it cannot be guaranteed that the system will converge to $\alpha \cdot \overrightarrow{1}$, where $\overrightarrow{1}=(1,1, \ldots, 1)^{T} \in \mathbb{R}^{n}$ and $\alpha$ is defined as above, i.e., reach consensus. On the other hand, if the graph stays connected during the whole process, then it has been shown that the agents will finally reach consensus. Since the control protocol is fixed in this problem, the initial position indeed determines the whole process. So a natural question is "under what initial condition will the agents reach consensus?".

\section{NON-SMOOTH MODEL}

Consider the standard Hegselmann-Krause model:

$$
\dot{x}_{i}(t)=\sum_{j:\left|x_{j}-x_{i}\right| \leq d}\left(x_{j}(t)-x_{i}(t)\right) .
$$

Although we have a discontinuous function on the righthand side, the almost surely existence and convergence of the solution to this differential equation have been proved in [9]. Here the convergence means the state will converge to an equilibrium of the system. However, the equilibrium is not necessarily in the form $\alpha \cdot \overrightarrow{1}$ as discussed before. Instead it can form several clusters [9].

\section{A. Sufficient Condition for consensus}

In this section, we give a sufficient condition on the initial states (opinions) such that the system will converge to exact one cluster. The concept of common neighbor will be used in the theorem.

Definition 3.1: For an undirected graph $G=(V, E)$, the set of common neighbors between two nodes $i$ and $j$ is defined as:

$$
\mathscr{N}_{i j}=\{k \in V \mid(i, k) \in E,(j, k) \in E\}=\mathscr{N}_{i} \cap \mathscr{N}_{j} .
$$

Theorem 3.2: For an initial condition $x(0) \in \mathbb{R}^{n}$ and the corresponding graph $G=(V, E)$, if $G$ is connected and for any pair $(i, j) \in E$, it holds that $\left|\mathscr{N}_{i j}\right| \geq \frac{n}{2}-2$, then the solution to (4) will converge to $\alpha \cdot \overrightarrow{1}$, where $\alpha=\frac{1}{n} \overrightarrow{1}^{T} x(0)$.

Proof: Because the initial graph $G$ is connected by assumption, if no edge in $E$ is lost during the process, the graph $G(t)$ will be always connected. Then it is well-known that the states will converge to the average value of the initial states.

What we need to show now is that for any pair of vertices $(i, j)$, the distance $\left|x_{j}(t)-x_{i}(t)\right|$ will not exceed the threshold $d$. Due to the continuity of $x(t)$, we consider the situation that $\left|x_{j}(t)-x_{i}(t)\right|=d$, and assume that $x_{j}(t)>x_{i}(t)$ without loss of generality. Denote $\mathscr{N}_{i}^{\prime}=\mathscr{N}_{i} \backslash \mathscr{N}_{i j}$ and $\mathscr{N}_{j}^{\prime}=\mathscr{N}_{j} \backslash \mathscr{N}_{i j}$.

$$
\begin{aligned}
& \frac{\mathrm{d}}{\mathrm{d} t}\left(x_{j}(t)-x_{i}(t)\right)=\left(\dot{x}_{j}(t)-\dot{x}_{i}(t)\right) \\
= & \sum_{k \in \mathscr{N}_{j}}\left(x_{k}(t)-x_{j}(t)\right)-\sum_{k \in \mathscr{N}_{i}}\left(x_{k}(t)-x_{i}(t)\right) \\
= & \sum_{k \in \mathscr{N}_{j}^{\prime}}\left(x_{k}(t)-x_{j}(t)\right)-\sum_{k \in \mathscr{N}_{i}^{\prime}}\left(x_{k}(t)-x_{i}(t)\right) \\
& -\left(\left|\mathscr{N}_{i j}\right|+2\right)\left(x_{j}(t)-x_{i}(t)\right) \\
\leq & \sum_{k \in \mathscr{N}_{j}^{\prime}}\left|x_{k}(t)-x_{j}(t)\right|+\sum_{k \in \mathscr{N}_{i}^{\prime}}\left|x_{k}(t)-x_{i}(t)\right| \\
& -\left(\left|\mathscr{N}_{i j}\right|+2\right) d \\
\leq & \left(\left|\mathscr{N}_{j}^{\prime}\right|+\left|\mathscr{N}_{i}^{\prime}\right|\right) d-\left(\left|\mathscr{N}_{i j}\right|+2\right) d . \\
\leq & \left(n-\left(\left|\mathscr{N}_{i j}+2\right|\right)\right) d-\left(\left|\mathscr{N}_{i j}\right|+2\right) d \\
\leq & \left(n-2\left(\left|\mathscr{N}_{i j}\right|+2\right)\right) d \\
\leq & 0 .
\end{aligned}
$$


As we can see, the distance $x_{j}(t)-x_{i}(t)$ will not increase in this case, which proves that the edge $(i, j)$ will not break if $\left|\mathscr{N}_{i j}\right| \geq \frac{n}{2}-2$ at time $t$.

Now suppose the first edge break happens right after time $t$ for the edge $(i, j)$. This means $\left|\mathscr{N}_{i j}\right|<\frac{n}{2}-2$ at time $t$. With the initial condition that $\left|\mathscr{N}_{i j}\right| \geq \frac{n}{2}-2$, the number of common neighbors of $\left|\mathscr{N}_{i j}\right|$ must have decreased at some time before $t$. But this can never happen without an edge break, which contradicts with the assumption that $(i, j)$ is the first edge to break. Therefore, no edge will break under the stated assumption. This concludes the proof.

Remark: The condition in Theorem 3.2 is not a necessary condition for reaching consensus. This can be easily shown by counter examples. Although it is weaker than the condition of a complete graph, it is still strong. With the constraint that edges are defined by distance, most vectors $x(0) \in \mathbb{R}^{n}$ do not satisfy this condition. If we consider infinitely many agents uniformly distributed on an interval of length $L$, then $L \leq 2 d$ is required in Theorem 3.2.

\section{B. Weighted Model}

As mentioned above, Theorem 3.2 holds for a limited number of initial conditions. Instead of finding a condition on the initial states, we modify the model slightly to guarantee opinion consensus for any $x(0)$ with a corresponding connected graph. Consider the following model:

$$
\dot{x}_{i}(t)=\sum_{j \in \mathscr{N}_{i}} \frac{1}{\left|\mathscr{N}_{i j}\right|+1}\left(x_{j}(t)-x_{i}(t)\right) .
$$

Since a discontinuous weight is added to the right-hand side of the already discontinuous H-K model (4), it is not obvious that the following statement, which is vital for the proof of our main theorem later, is true.

Proposition 3.3: Given an initial condition $x(0) \in \mathbb{R}^{n}$ satisfying $x_{i}(0) \neq x_{j}(0)$ for any $i \neq j$, we have $x_{i}(T) \neq x_{j}(T)$ for any $i \neq j$ and any $T \in[0, \infty)$, where $x(t)$ is the solution to the differential equation (6).

Proof: We will prove the proposition by showing that the distance between any pair of nodes is larger than the value of some exponential function which will not reach zero in finite time. This is not clearly to be true since the weight defined by the number of common neighbors may be different between different pairs of nodes.

Without loss of generality, we only need to investigate the distance between two adjacent agents $i$ and $j$, where $j$ is to the right of $i$. Suppose at time $t \geq 0$, the distance between $i$ and $j$ is $x_{j}(t)-x_{i}(t)=\delta$ for some $0<\delta \leq$ d. Define $\mathscr{N}_{j}^{\mathrm{f}}=\left\{k \in \mathscr{N}_{j} \mid x_{k}(t)-x_{i}(t)>d\right\}, \mathscr{N}_{j}^{\mathrm{c}}=\{k \in$ $\left.\mathscr{N}_{j} \mid 0<x_{k}(t)-x_{i}(t) \leq d\right\}, \mathscr{N}_{i}^{\mathrm{f}}=\left\{k \in \mathscr{N}_{i} \mid x_{j}(t)-x_{k}(t)>d\right\}$ and $\mathscr{N}_{i}^{\mathrm{c}}=\left\{k \in \mathscr{N}_{i} \mid 0<x_{j}(t)-x_{k}(t) \leq d\right\}$. Fig 1 gives a visualization of these definitions. It is not hard to prove the following facts:

(i) $\mathscr{N}_{i}=\mathscr{N}_{i}^{\mathrm{f}} \cup \mathscr{N}_{i}^{\mathrm{c}} \cup \mathscr{N}_{j}^{\mathrm{c}} \cup\{j\}$;

(ii) $\mathscr{N}_{j}=\mathscr{N}_{j}^{\mathrm{f}} \cup \mathscr{N}_{j}^{\mathrm{c}} \cup \mathscr{N}_{i}^{\mathrm{c}} \cup\{i\}$;

(iii) for any $k \in \mathscr{N}_{i}^{\mathrm{f}},\left|\mathscr{N}_{i k}\right|=\left|\mathscr{N}_{i}^{\mathrm{f}}\right|+\left|\mathscr{N}_{i}^{\mathrm{c}}\right|-1$;

(iv) for any $k \in \mathscr{N}_{j}^{\mathrm{f}},\left|\mathscr{N}_{j k}\right|=\left|\mathscr{N}_{j}^{\mathrm{f}}\right|+\left|\mathscr{N}_{j}^{\mathrm{c}}\right|-1$;

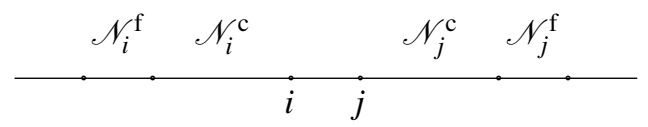

Fig. 1. A graphic illustration of the sets $\mathscr{N}_{i}^{\mathrm{f}}, \mathscr{N}_{i}^{\mathrm{c}}, \mathscr{N}_{j}^{\mathrm{c}}, \mathscr{N}_{j}^{\mathrm{f}}$

(v) for any $k \in \mathscr{N}_{i}^{\mathrm{c}},\left|\mathscr{N}_{i k}\right|=\left|\mathscr{N}_{j k}\right|+\left|\mathscr{N}_{i}^{\mathrm{f}}\right|$;

(vi) for any $k \in \mathscr{N}_{j}^{\mathrm{c}},\left|\mathscr{N}_{j k}\right|=\left|\mathscr{N}_{i k}\right|+\left|\mathscr{N}_{j}^{\mathrm{f}}\right|$.

For example (iv) is true since for $k \in \mathscr{N}_{j}^{\mathrm{f}}$,

$$
\begin{aligned}
\mathscr{N}_{j k} & =\mathscr{N}_{j} \cap \mathscr{N}_{k}=\left(\mathscr{N}_{j}^{\mathrm{f}} \cup \mathscr{N}_{j}^{\mathrm{c}} \cup \mathscr{N}_{i}^{\mathrm{c}} \cup\{i\}\right) \cap \mathscr{N}_{k} \\
& =\left(\left(\mathscr{N}_{j}^{\mathrm{f}} \cup \mathscr{N}_{j}^{\mathrm{c}}\right) \cap \mathscr{N}_{k}\right) \cup\left(\left(\mathscr{N}_{i}^{\mathrm{c}} \cup\{i\}\right) \cap \mathscr{N}_{k}\right) \\
& =\left(\mathscr{N}_{j}^{\mathrm{f}} \cup \mathscr{N}_{j}^{\mathrm{c}} \backslash\{k\}\right) \cup \phi \\
& =\mathscr{N}_{j}^{\mathrm{f}} \cup \mathscr{N}_{j}^{\mathrm{c}} \backslash\{k\},
\end{aligned}
$$

and (vi) is true since for $k \in \mathscr{N}_{j}^{\mathrm{c}}$,

$$
\begin{aligned}
\mathscr{N}_{i k} \cup \mathscr{N}_{j}^{\mathrm{f}} & =\left(\mathscr{N}_{i} \cap \mathscr{N}_{k}\right) \cup \mathscr{N}_{j}^{\mathrm{f}} \\
& =\left(\left(\mathscr{N}_{i}^{\mathrm{f}} \cup \mathscr{N}_{i}^{\mathrm{c}} \cup \mathscr{N}_{j}^{\mathrm{c}} \cup\{j\}\right) \cap \mathscr{N}_{k}\right) \cup \mathscr{N}_{j}^{\mathrm{f}} \\
& =\left(\left(\mathscr{N}_{i}^{\mathrm{f}} \cap \mathscr{N}_{k}\right) \cup\left(\left(\mathscr{N}_{i}^{\mathrm{c}} \cup \mathscr{N}_{j}^{\mathrm{c}} \cup\{j\}\right) \cap \mathscr{N}_{k}\right)\right) \cup \mathscr{N}_{j}^{\mathrm{f}} \\
& =\left(\left(\mathscr{N}_{i}^{\mathrm{c}} \cup \mathscr{N}_{j}^{\mathrm{c}} \cup\{j\}\right) \cap \mathscr{N}_{k}\right) \cup \mathscr{N}_{j}^{\mathrm{f}} \\
& =\left(\left(\mathscr{N}_{i}^{\mathrm{c}} \cup \mathscr{N}_{j}^{\mathrm{c}} \cup\{j\}\right) \cup \mathscr{N}_{j}^{\mathrm{f}}\right) \cap\left(\mathscr{N}_{k} \cup \mathscr{N}_{j}^{\mathrm{f}}\right) \\
& =\left(\mathscr{N}_{j} \cup\{j\} \backslash\{i\}\right) \cap \mathscr{N}_{k} \\
& =\left(\mathscr{N}_{j} \cap \mathscr{N}_{k}\right) \cup\{j\} \backslash\{i\} \\
& =\mathscr{N}_{j k} \cup\{j\} \backslash\{i\} .
\end{aligned}
$$

Consider the derivative of the difference $x_{j}(t)-x_{i}(t)$ :

$$
\begin{aligned}
& \frac{\mathrm{d}}{\mathrm{d} t}\left(x_{j}(t)-x_{i}(t)\right)=\dot{x}_{j}(t)-\dot{x}_{i}(t) \\
= & \sum_{k \in \mathscr{N}_{j}} \frac{x_{k}(t)-x_{j}(t)}{\left|\mathscr{N}_{j k}\right|+1}-\sum_{k \in \mathscr{N}_{i}} \frac{x_{k}(t)-x_{i}(t)}{\left|\mathscr{N}_{i k}\right|+1} \\
= & \sum_{k \in \mathscr{N}_{j}^{\mathrm{f}}} \frac{x_{k}(t)-x_{j}(t)}{\left|\mathscr{N}_{j k}\right|+1}+\sum_{k \in \mathscr{N}_{j}^{\mathrm{c}}}\left(\frac{x_{k}(t)-x_{j}(t)}{\left|\mathscr{N}_{j k}\right|+1}-\frac{x_{k}(t)-x_{i}(t)}{\left|\mathscr{N}_{i k}\right|+1}\right) \\
& -\sum_{k \in \mathscr{N}_{i}^{\mathrm{f}}} \frac{x_{k}(t)-x_{i}(t)}{\left|\mathscr{N}_{i k}\right|+1}+\sum_{k \in \mathscr{N}_{i}^{\mathrm{c}}}\left(\frac{x_{k}(t)-x_{j}(t)}{\left|\mathscr{N}_{j k}\right|+1}-\frac{x_{k}(t)-x_{i}(t)}{\left|\mathscr{N}_{i k}\right|+1}\right) \\
& +\frac{2}{\left|\mathscr{N}_{i j}\right|+1}\left(x_{i}(t)-x_{j}(t)\right) .
\end{aligned}
$$

For $k \in \mathscr{N}_{j}^{\mathrm{c}}$, we have

$$
\begin{aligned}
& \frac{1}{\left|\mathscr{N}_{j k}\right|+1}\left(x_{k}(t)-x_{j}(t)\right)-\frac{1}{\left|\mathscr{N}_{i k}\right|+1}\left(x_{k}(t)-x_{i}(t)\right) \\
= & \frac{1}{\left|\mathscr{N}_{i k}\right|+1}\left(x_{k}(t)-x_{j}(t)\right)-\frac{1}{\left|\mathscr{N}_{i k}\right|+1}\left(x_{k}(t)-x_{i}(t)\right) \\
& +\frac{1}{\left|\mathscr{N}_{j k}\right|+1}\left(x_{k}(t)-x_{j}(t)\right)-\frac{1}{\left|\mathscr{N}_{i k}\right|+1}\left(x_{k}(t)-x_{j}(t)\right) \\
= & \frac{1}{\left|\mathscr{N}_{i k}\right|+1}\left(x_{i}(t)-x_{j}(t)\right) \\
& +\frac{\left|\mathscr{N}_{i k}\right|-\left|\mathscr{N}_{j k}\right|}{\left(\left|\mathscr{N}_{j k}\right|+1\right)\left(\left|\mathscr{N}_{i k}\right|+1\right)}\left(x_{k}(t)-x_{j}(t)\right)
\end{aligned}
$$




$$
\begin{aligned}
= & \frac{1}{\left|\mathscr{N}_{i k}\right|+1}\left(x_{i}(t)-x_{j}(t)\right) \\
& -\frac{\left|\mathscr{N}_{j}^{\mathrm{f}}\right|}{\left(\left|\mathscr{N}_{j k}\right|+1\right)\left(\left|\mathscr{N}_{i k}\right|+1\right)}\left(x_{k}(t)-x_{j}(t)\right) \\
\geq & \left(x_{i}(t)-x_{j}(t)\right)-\frac{\left|\mathscr{N}_{j}^{\mathrm{f}}\right|}{\left(\left|\mathscr{N}_{j}^{\mathrm{f}}\right|+\left|\mathscr{N}_{j}^{\mathrm{c}}\right|\right)\left|\mathscr{N}_{j}^{\mathrm{c}}\right|}(d-\delta) .
\end{aligned}
$$

The last equality holds since $\left|\mathscr{N}_{j k}\right|-\left|\mathscr{N}_{i k}\right|=\left|\mathscr{N}_{j}^{\mathrm{f}}\right|$ by (vi), and the last inequality is true because $\mathscr{N}_{j}^{\mathrm{f}} \cup \mathscr{N}_{j}^{\mathrm{c}} \subset \mathscr{N}_{j k} \cup\{k\}$, $\mathscr{N}_{j}^{\mathrm{c}} \subset \mathscr{N}_{i k} \cup\{k\}$ and $x_{k}(t)-x_{j}(t) \leq d-\delta$.

If we sum up over $k \in \mathscr{N}_{j}^{\mathrm{c}}$, we get

$$
\begin{aligned}
& \sum_{k \in \mathscr{N}_{j}^{\mathrm{c}}}\left(\frac{1}{\left|\mathscr{N}_{j k}\right|+1}\left(x_{k}(t)-x_{j}(t)\right)-\frac{1}{\left|\mathscr{N}_{i k}\right|+1}\left(x_{k}(t)-x_{i}(t)\right)\right) \\
\geq & \left|\mathscr{N}_{j}^{\mathrm{c}}\right|\left(\left(x_{i}(t)-x_{j}(t)\right)-\frac{\left|\mathscr{N}_{j}^{\mathrm{f}}\right|}{\left(\left|\mathscr{N}_{j}^{\mathrm{f}}\right|+\left|\mathscr{N}_{j}^{\mathrm{c}}\right|\right)\left|\mathscr{N}_{j}^{\mathrm{c}}\right|}(d-\delta)\right) \\
= & \left|\mathscr{N}_{j}^{\mathrm{c}}\right|\left(x_{i}(t)-x_{j}(t)\right)-\frac{\left|\mathscr{N}_{j}^{\mathrm{f}}\right|}{\left|\mathscr{N}_{j}^{\mathrm{f}}\right|+\left|\mathscr{N}_{j}^{\mathrm{c}}\right|}(d-\delta) .
\end{aligned}
$$

For $k \in \mathscr{N}_{j}^{\mathrm{f}}$, we have

$$
\begin{aligned}
& \sum_{k \in \mathscr{N}_{j}^{\mathrm{f}}} \frac{1}{\left|\mathscr{N}_{j k}\right|+1}\left(x_{k}(t)-x_{j}(t)\right) \\
= & \sum_{k \in \mathscr{N}_{j}^{\mathrm{f}}} \frac{1}{\left|\mathscr{N}_{j}^{\mathrm{f}}\right|+\left|\mathscr{N}_{j}^{\mathrm{c}}\right|}\left(x_{k}(t)-x_{j}(t)\right) \\
\geq & \sum_{k \in \mathscr{N}_{j}^{\mathrm{f}}} \frac{1}{\left|\mathscr{N}_{j}^{\mathrm{f}}\right|+\left|\mathscr{N}_{j}^{\mathrm{c}}\right|}(d-\delta) \\
= & \frac{\left|\mathscr{N}_{j}^{\mathrm{f}}\right|}{\left|\mathscr{N}_{j}^{\mathrm{f}}\right|+\left|\mathscr{N}_{j}^{\mathrm{c}}\right|}(d-\delta) .
\end{aligned}
$$

One can get similar results for $k \in \mathscr{N}_{i}^{\mathrm{c}}$ and $k \in \mathscr{N}_{i}^{\mathrm{f}}$. When plugging all these results to (7), we get

$$
\begin{aligned}
& \frac{\mathrm{d}}{\mathrm{d} t}\left(x_{j}(t)-x_{i}(t)\right)=\dot{x}_{j}(t)-\dot{x}_{i}(t) \\
\geq & \frac{\left|\mathscr{N}_{j}^{\mathrm{f}}\right|}{\left|\mathscr{N}_{j}^{\mathrm{f}}\right|+\left|\mathscr{N}_{j}^{\mathrm{c}}\right|}(d-\delta)+\frac{\left|\mathscr{N}_{i}^{\mathrm{f}}\right|}{\left|\mathscr{N}_{i}^{\mathrm{f}}\right|+\left|\mathscr{N}_{i}^{\mathrm{c}}\right|}(d-\delta) \\
& +\left|\mathscr{N}_{j}^{\mathrm{c}}\right|\left(x_{i}(t)-x_{j}(t)\right)-\frac{\left|\mathscr{N}_{j}^{\mathrm{f}}\right|}{\left|\mathscr{N}_{j}^{\mathrm{f}}\right|+\left|\mathscr{N}_{j}^{\mathrm{c}}\right|}(d-\delta) \\
& +\left|\mathscr{N}_{i}^{\mathrm{c}}\right|\left(x_{i}(t)-x_{j}(t)\right)-\frac{\left|\mathscr{N}_{i}^{\mathrm{f}}\right|}{\left|\mathscr{N}_{i}^{\mathrm{f}}\right|+\left|\mathscr{N}_{i}^{\mathrm{c}}\right|}(d-\delta) \\
& +\frac{2}{\left|\mathscr{N}_{i j}\right|+1}\left(x_{i}(t)-x_{j}(t)\right) \\
\geq & \left(\left|\mathscr{N}_{i}^{\mathrm{c}}\right|+\left|\mathscr{N}_{j}^{\mathrm{c}}\right|+2\right)\left(x_{i}(t)-x_{j}(t)\right) \\
\geq & n\left(x_{i}(t)-x_{j}(t)\right)=-n\left(x_{j}(t)-x_{i}(t)\right) .
\end{aligned}
$$

In fact, (8) holds for all $t$ such that $0<x_{j}(t)-x_{i}(t) \leq d$. We know that the solution to the differential equation: $\dot{y}(t)=$ $-n y(t)$ is $y(t)=y(0) \mathrm{e}^{-n t}$. If we denote $d_{i j}(t)=x_{j}(t)-x_{i}(t)$, then $\dot{d}_{i j}(t) \geq-n d_{i j}(t)$. In other words, $d_{i j}(t) \geq d_{i j}(0) \mathrm{e}^{-n t}>$ 0 , since $d_{i j}(0)>0$. Thus, $x_{i}(T) \neq x_{j}(T)$ for all $T \in[0, \infty)$.
We will also use the following concept from graph theory:

Definition 3.4: For an undirected graph $G=(V, E)$, an edge $(i, j) \in E$ is called crucial if $\mathscr{N}_{i j}=\phi$, i.e. there does not exist $k \in V$ such that $(i, k) \in E$ and $(k, j) \in E$ simultaneously.

Theorem 3.5: (Main Theorem) For any initial condition $x(0) \in \mathbb{R}^{n}$ such that:

- the corresponding graph is connected;

- $x_{i}(0) \neq x_{j}(0)$ for any $i \neq j$,

the system (6) will converge to the equilibrium $\alpha \cdot \overrightarrow{\mathbf{1}}$, where $\alpha=\frac{1}{n} \overrightarrow{1}^{T} x(0)$, i.e., consensus is reached.

Proof: If no crucial edge breaks during the process, the graph will keep connected. Therefore, we just need to check when a crucial edge is going to break, i.e., $\left|x_{i}(t)-x_{j}(t)\right|=d$, and $\mathscr{N}_{i j}=\phi$. Assuming that $x_{j}(t)>x_{i}(t)$ without loss of generality, we get

$$
\begin{aligned}
& \frac{\mathrm{d}}{\mathrm{d} t}\left(x_{j}(t)-x_{i}(t)\right)=\dot{x}_{j}(t)-\dot{x}_{i}(t) \\
= & \sum_{k \in \mathscr{N}_{j}} \frac{x_{k}(t)-x_{j}(t)}{\left|\mathscr{N}_{k j}\right|+1}-\sum_{k \in \mathscr{N}_{i}} \frac{x_{k}(t)-x_{i}(t)}{\left|\mathscr{N}_{k i}\right|+1} \\
= & \sum_{k \in \mathscr{N}_{j} \backslash\{i\}} \frac{1}{\left|\mathscr{N}_{k j}\right|+1}\left(x_{k}(t)-x_{j}(t)\right) \\
& -\sum_{k \in \mathscr{N}_{i} \backslash\{j\}} \frac{1}{\left|\mathscr{N}_{k i}\right|+1}\left(x_{k}(t)-x_{i}(t)\right)-2\left(x_{j}(t)-x_{i}(t)\right) \\
\leq & \left(\sum_{k \in \mathscr{N}_{j} \backslash\{i\}} \frac{1}{\left|\mathscr{N}_{k j}\right|+1}+\sum_{k \in \mathscr{N}_{i} \backslash\{j\}} \frac{1}{\left|\mathscr{N}_{k i}\right|+1}-2\right) d .
\end{aligned}
$$

Now we only need to show that

$$
\sum_{k \in \mathscr{N}_{j} \backslash\{i\}} \frac{1}{\left|\mathscr{N}_{k j}\right|+1}+\sum_{k \in \mathscr{N}_{i} \backslash\{j\}} \frac{1}{\left|\mathscr{N}_{k i}\right|+1} \leq 2
$$

in order to prove $\frac{d}{d t}\left(x_{j}(t)-x_{i}(t)\right) \leq 0$. We will now show that for all $k \in \mathscr{N}_{j} \backslash\{i\}$, we have that $\left|\mathscr{N}_{k j}\right|=\left|\mathscr{N}_{j}\right|-2$, if $\left|\mathscr{N}_{j}\right| \geq 2$.

Due to the assumption we have made, there is no pair of agents with the same initial opinion. According to Proposition 3.3, there will not be two agents reaching the same state in finite time. So we have $x_{i}(t) \neq x_{j}(t)$ for any $i \neq j$ and $t<$ $\infty$. If $(i, j)$ is a crucial edge with $x_{j}(t)-x_{i}(t)=d$, then agent $j$ has only one neighbor to its left which is agent $i$. Then all the other neighbors of $j$ must be located to its right. If $\left|\mathscr{N}_{j}\right| \geq 2$, every $j$ 's right neighbor $j^{\prime}$ is a common neighbor of $j$ and another right neighbor $j^{\prime \prime}$. This is because $\left|x_{j^{\prime}}(t)-x_{j}(t)\right| \leq d$ and $\left|x_{j^{\prime}}(t)-x_{j^{\prime \prime}}(t)\right| \leq \max \left\{x_{j^{\prime}}(t)-x_{j}(t), x_{j^{\prime \prime}}(t)-x_{j}(t)\right\} \leq d$. We have $i \notin \mathscr{N}_{k j}$ according to the definition of a crucial edge. Therefore, we have $\mathscr{N}_{k j}=\mathscr{N}_{j} \backslash\{i, k\}$, which implies $\left|\mathscr{N}_{k j}\right|=\left|\mathscr{N}_{j}\right|-2$ for $k \in \mathscr{N}_{j} \backslash\{i\}$. Equivalently one can get $\left|\mathscr{N}_{k i}\right|=\left|\mathscr{N}_{i}\right|-2$ for $k \in \mathscr{N}_{i} \backslash\{j\}$ if $\left|\mathscr{N}_{i}\right| \geq 2$.

By plugging these results into the left-hand side of the 
inequality (9), we get

$$
\begin{aligned}
& \sum_{k \in \mathscr{N}_{j} \backslash\{i\}} \frac{1}{\left|\mathscr{N}_{k j}\right|+1}+\sum_{k \in \mathscr{N}_{i} \backslash\{j\}} \frac{1}{\left|\mathscr{N}_{k i}\right|+1} \\
= & \sum_{k \in \mathscr{N}_{j} \backslash\{i\}} \frac{1}{\left|\mathscr{N}_{j}\right|-1}+\sum_{k \in \mathscr{N}_{i} \backslash\{j\}} \frac{1}{\left|\mathscr{N}_{i}\right|-1} \\
= & \frac{\left|\mathscr{N}_{j}\right|-1}{\left|\mathscr{N}_{j}\right|-1}+\frac{\left|\mathscr{N}_{i}\right|-1}{\left|\mathscr{N}_{i}\right|-1}=2 .
\end{aligned}
$$

Note that if $\left|\mathscr{N}_{j}\right|=1$, which means $\mathscr{N}_{j}=\{i\}$ and $\mathscr{N}_{j} \backslash\{i\}=\phi$, then (9) is also true since the first term on the left-hand side is equal to 0 .

However, in Theorem 3.5, it is required that no two agents have the exact same initial opinion. Although this is a set of measure zero in the state space, it is a common scenario in reality. To accommodate this scenario and at the same time avoid some numerical difficulties encountered when the agents are very close to each other, one can treat all the agents with the same state as one new agent. This is equivalent to saying that we still treat agents with the same state separately but with a weight divided by the number of agents at that position. If $M_{j}$ is defined as the number of agents which have the same position as agent $j$, then (6) can be rewritten as:

$$
\dot{x}_{i}(t)=\sum_{j \in \mathscr{N}_{i}} \frac{1}{\left(\left|\tilde{\mathscr{N}}_{i j}\right|+1\right) M_{j}}\left(x_{j}(t)-x_{i}(t)\right),
$$

where $\left|\tilde{N}_{i j}\right|$ is the number of common opinion clusters between $i$ and $j$. Here two agents belong to the same cluster if and only if they have the same opinion. We can show that $\left|\tilde{\mathscr{N}}_{i j}\right|=\sum_{k \in \mathscr{N}_{i j} \cup\{i, j\}} 1 / M_{k}-2$. It is not hard to prove the following corollary:

Corollary 3.6: For any initial condition $x(0) \in \mathbb{R}^{n}$ whose corresponding graph is connected, the control protocol (10) will guarantee consensus.

Nevertheless, on the right-hand side of (10) the weight is not symmetric. So in Corollary 3.6 the equilibrium is actually not the initial average. This is in some sense a shortcoming since we ignore the weight of those agents who have the same opinion.

\section{SMOOTHED MODEL}

Another issue for the original H-K model (4) is the discontinuous right-hand side. In the theory of differential equations, the condition of Lipschitz continuity is essential for the existence and uniqueness of the solution. As stated in [9], the convergence of the solution to (4) is guaranteed for almost all initial conditions, which implies there can exist a set (with measure zero) of singular points. Moreover, some numerical problems may arise from this discontinuity when one wants to implement the model. For example, the relative error will be unbounded if the distance is around the threshold $d$ in the H-K model.

A common remedy for these problems is to approximate the original function by a continuous (even differentiable in some cases) function. The approximation has the same value as the original function except around the points where the discontinuity occurs. Around those points, a smoothing function is used to replace the original function, e.g., [5].

We rewrite the original model as:

$$
\dot{x}_{i}(t)=\sum_{j \neq i} \rho_{i j}\left(x_{j}(t)-x_{i}(t)\right)
$$

where

$$
\rho_{i j}= \begin{cases}1, & \left|x_{i}(t)-x_{j}(t)\right| \leq d \\ 0, & \left|x_{i}(t)-x_{j}(t)\right|>d\end{cases}
$$

We can modify $\rho_{i j}(x)$ in the following way: we denote by $\beta_{i j}=\left|x_{i}-x_{j}\right|^{2}$ the distance between agent $i$ and agent $j$ and introduce a potential function between $i$ and $j$ as:

$$
r\left(\beta_{i j}\right)=\left\{\begin{array}{lc}
\beta_{i j}, & 0 \leq \beta_{i j} \leq d^{2}, \\
\varphi\left(\beta_{i j}\right), & d^{2}<\beta_{i j} \leq(d+\varepsilon)^{2}, \\
c, & (d+\varepsilon)^{2}<\beta_{i j}<\infty .
\end{array}\right.
$$

where $c$ is a positive constant and $\varphi$ is a chosen monotonically increasing function on the interval $\left[\left(d^{2}, d+\varepsilon\right)^{2}\right]$ to make $r\left(\beta_{i j}\right)$ differentiable for any $\beta_{i j} \in(0, \infty)$ (e.g., high order polynomials). Then let

$$
\begin{aligned}
\rho_{i j} & =\frac{\partial r\left(\beta_{i j}\right)}{\partial \beta_{i j}} \\
& =\left\{\begin{array}{lr}
1, & 0 \leq \beta_{i j} \leq d^{2}, \\
\varphi^{\prime}\left(\beta_{i j}\right), & d^{2}<\beta_{i j} \leq(d+\varepsilon)^{2}, \\
0, & (d+\varepsilon)^{2}<\beta_{i j}<\infty .
\end{array}\right.
\end{aligned}
$$

\section{A. Convergence}

If we consider the model (11) with the choice of $\rho_{i j}$ in (13), then the right-hand side is a Lipschitz continuous function, which will ensure the existence and the uniqueness of the solution to the differential equation. But convergence may not be guaranteed in this case. Therefore, the following theorem is required.

Theorem 4.1: For any initial condition $x(0) \in \mathbb{R}^{n}$, there exists a vector $x^{*} \in \mathbb{R}^{n}$ such that the solution $x(t)$ to the differential equation (11) with the choice of $\rho_{i j}$ in (13) will converge to $x^{*}$ as $t \rightarrow \infty$.

Remark: The main idea of the proof is to use LaSalle's invariance principle. We skip the proof here duo to the space limitation. However, one can easily figure out that for the equilibrium $x^{*}$ of the system, either $x_{i}^{*}=x_{j}^{*}$ or $\left|x_{i}^{*}-x_{j}^{*}\right| \geq$ $d+\varepsilon$ holds. Both situation may occur in the largest invariant subset when one applies LaSalle's invariance principle. It is the reason why more than one clusters may occur for some initial states.

\section{B. Sufficient Condition for consensus}

Similarly to section 3.1 , there is a sufficient condition for the initial states to guarantee consensus by using the control protocol (11). When the smoothed model (11) is used, the weight between a pair of nodes $(i, j)$ continuously decreases to zero when the distance exceeds $d$. Although one considers $(i, j)$ to be an edge when there is a positive weight on it in general, we will stick on the previous definition of the edge set, which is $E=\left\{(i, j)|| x_{i}-x_{j} \mid \leq d\right\}$. Then we will get the smoothed version of Theorem 3.2. 


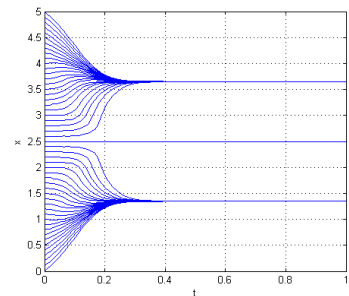

(a)

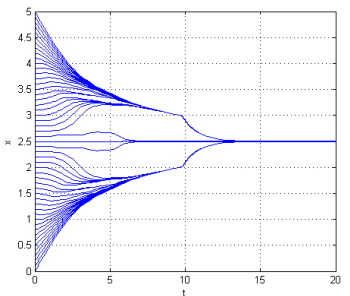

(b)
Fig. 2. Time evolution of 51 agent opinions according to (case (a)) model (4) and (case (b)) model (6). Initial opinions are uniformly spaced on an interval of length 5. The interaction radius $d$ is chosen to be 0.98 .

Theorem 4.2: For an initial condition $x(0) \in \mathbb{R}^{n}$, if the corresponding graph $G=(V, E)$ is connected and for any pair $(i, j) \in E,\left|\mathscr{N}_{i j}\right| \geq \frac{n}{2}-2$, then the solution to (11) will converge to $\alpha \cdot \overrightarrow{1}$, where $\alpha=\frac{1}{n} \overrightarrow{1}^{T} x(0)$.

Proof: The proof is similar to that of Theorem 3.2 and is thus omitted.

\section{Simulations}

We will present some simulation results of the weighted non-smooth models in this section.

In the first example, 51 agent opinions are initially uniformly spaced on an interval of length 5 . The interaction radius $d$ is chosen to be 0.98 to avoid some singularity from the discontinuous right-hand side in the non-smooth models. We use both the original H-K model (4) and the weighted model (6). Fig. 2 shows the simulation result. The original H$\mathrm{K}$ model diverges to three clusters (in (a)) and the modified model (6) reaches consensus (in (b)).

In the second example, we want to show how the coincidence of initial opinions affects the simulation result by using the two modified models: (6) and (10). 26 agent opinions are uniformly spread on the interval of length 5 , while the other 20 opinions are all located at position 1 initially. $d$ is chosen to be 0.98 again. The initial opinion average $\alpha$ is approximately 1.85 . Although the initial distribution does not fulfill the condition in Theorem 3.5, the system does converge to the initial opinion average by using the control protocol (6) (in Fig. 3(a)). If only the opinion cluster is considered, these 20 agent opinions is in some sense ignored since there is also one agent opinion positioned at 1 among the first 26 agent opinions. So using model (10), we get a symmetric result in Fig. 3(b), and the compromised opinion is 2 in the end.

\section{CONClusions AND FUtURE WORK}

In this paper, we first gave a sufficient condition for opinions consensus for the original Hegselmann-Krause model, as well as for a model with a continuous right-hand side. Furthermore, we provided two modified versions of the Hegselmann-Krause model such that consensus is guaranteed for any initial configuration corresponding to a connected graph.

Future work will examine the case of higher dimensional spaces, and in particular the two dimensional space. It is not

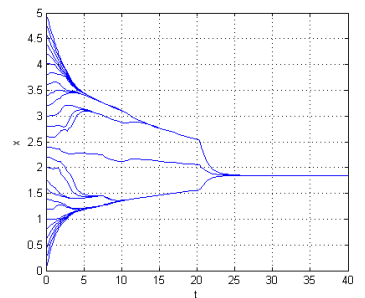

(a)

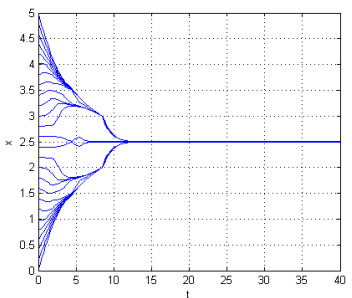

(b)
Fig. 3. Time evolution of 46 agent opinions according to (case (a)) model (6) and (case (b)) model (10). Initial opinions of 26 agents are uniformly spaced on an interval of length 5 , while the rest 20 agents are all initially positioned at 1 . The interaction radius $d$ is chosen to be 0.98 .

hard to see that Theorem 3.2, 4.1, and 4.2 can be extended to any finite dimensional spaces $\mathbb{R}^{m}$ if the absolute value is modified to the Euclidean norm, and with an appropriate use of the Kronecker product. However, it should be noted that Theorem 3.5 is not extendable to the higher dimensional case in a straightforward manner since the line structure is used in the proof. So the extension to higher dimensions may require more effort in this case.

\section{REFERENCES}

[1] R. Hegselmann and U. Krause, "Opinion dynamics and bounded confidence models, analysis, and simulation", Journal of Artificial Societies and Social Simulation, vol. 5, no. 3, 2002.

[2] G. Weisbuch and G. Deffuant and F. Amblard and J. Nadal, "Meet, discuss, and segregate!", Complexity, vol. 7, no. 3, 2002, pp. 55-63.

[3] J. Lorenz, "Consensus strikes back in the Hegselmann-Krause model of continuous opinion dynamics under bounded confidence", Journal of Artificial Societies and Social Simulation, vol. 9, no. 1, pp. 8.

[4] J. Lorenz, "A stabilization theorem for dynamics of continuous opinions", Physica A: Statistical Mechanics and its Applications, vol. 355, issue 1, 2005, pp. 217-223.

[5] R. Olfati-Saber and R.M. Murray, "Flocking with obstacle avoidance: cooperation with limited communication in mobile networks", Proceedings of the 42nd IEEE Conference on Decision and Control, vol. 2, 2003, pp. 2022-2028.

[6] R. Olfati-Saber and R.M. Murray, "Consensus problems in networks of agents with switching topology and time-delays", IEEE Transactions on Automatic Control, vol. 49, issue 9, 2004, pp. 1520-1533.

[7] R. Olfati-Saber and R.M. Murray and J.A. Fax, "Consensus and Cooperation in Networked Multi-Agent Systems", Proceedings of the IEEE, vol. 95, no.1, 2007, pp. 215-233.

[8] V.D. Blondel and J.M. Hendrickx and J.N. Tsitsiklis , "On Krause's Multi-Agent Consensus Model With State-Dependent Connectivity", IEEE Transactions on Automatic Control, vol.54, no.11, 2009, pp. 2586-2597.

[9] V.D. Blondel and J.M. Hendrickx and J.N. Tsitsiklis, "Continuoustime average-preserving opinion dynamics with opinion-dependent communications", SIAM J. Control and Optimization, 2010, pp. 52145240.

[10] D.V. Dimarogonas and K.H. Johansson, "Decentralized connectivity maintenance in mobile networks with bounded inputs", , 2008 IEEE International Conference on Robotics and Automation, pp. 1507-1512.

[11] M.C. DeGennaro and A. Jadbabaie, "Decentralized control of connectivity for multi-agent systems", 45th IEEE Conf. Decision and Control, 2006, pp. 3628-3633.

[12] M. Ji and M. Egerstedt, "Connectedness preserving distibuted coordination control over dynamic graphs", 2005 American Control Conference, pp. 93-98.

[13] T. Gustavi and D.V. Dimarogonas and M. Egerstedt and X. Hu, "Sufficient conditions for connectivity maintenance and rendezvous in leader-follower networks", Automatica, vol. 46, issue 1, 2010, pp. 133-139. 